\title{
Chromosomal abnormalities among 246 fetuses with pleural effusions detected on prenatal ultrasound examination: Factors associated with an increased risk of aneuploidy
}

Kendra Waller, $M S^{1}$, Surasith Chaithongwongwatthana, $M D^{2}$, Waralak Yamasmit, $M D^{3}$, and Alan E. Donnenfeld, $M D^{4}$

\begin{abstract}
Purpose: To determine the prevalence of chromosomal abnormalities in fetuses with prenatally diagnosed pleural effusions and to identify factors associated with an increased risk of aneuploidy. Methods: A retrospective analysis of the Genzyme Genetics database was performed for samples submitted from October 1994 to April 2003 with an indication of fetal pleural effusion. Results: There were 246 samples in which pleural effusion was identified as an indication for prenatal chromosome analysis. Ninety-four were from fetuses with isolated pleural effusions and 152 had other abnormalities in addition to pleural effusion. The prevalence of chromosome abnormalities was 35.4\% (95\% confidence interval, 29.2-41.4\%). Among the eight first trimester samples, the aneuploidy rate was $63 \%$. Pleural effusion cases associated with additional sonographic findings had a significantly higher aneuploidy rate than the isolated pleural effusion cases ( $50 \%$ vs. $12 \%, P<0.001)$. Conclusions: Chromosome analysis is warranted after the prenatal detection of a fetal pleural effusion. The risk of aneuploidy is greater with first trimester detection and is significantly increased in the presence of other associated anomalies. Genet Med 2005:7(6): 417-421.
\end{abstract}

Key Words: pleural effusion, hydrops, aneuploidy, prenatal diagnosis, Turner syndrome

Fetal pleural effusions may occur as an isolated finding or in association with other abnormalities such as fetal hydrops, lung tumors, cardiac anomalies, arrhythmias, and other congenital defects. Primary pleural effusions are most often chylous and constitute the majority of isolated fetal pleural effusions. Secondary effusions most commonly result from nonimmune hydrops. The incidence of fetal pleural effusion has been estimated to be one in 15,000 pregnancies. ${ }^{1,2}$ However, a recent prospective study performed in low-risk obstetric cases at 7 to 10 weeks gestation showed that fetal pleural effusion may be found in as high as $1.2 \%$ of all pregnancies. ${ }^{3}$

The association of aneuploidy with prenatally diagnosed fetal pleural effusion is well known. ${ }^{2,4,5}$ In these reports, aneuploidy rates for second and third trimester fetuses with pleural effusions have ranged from $3.2 \%$ to $5.8 \%$. The risk of aneuploidy with first trimester diagnosis of pleural effusion is significantly greater. Hashimoto et al. identified chromosome abnormalities in 9 of $11(82 \%)$ first trimester embryos and fetuses

From Genzyme Genetics, ${ }^{1}$ Dallas, Texas and ${ }^{4}$ Philadelphia, Pennsylvania; ${ }^{4}$ Drexel University College of Medicine, Philadelphia, Pennsylvania; ${ }^{2}$ Chulalongkorn University and Bangkok Metropolitan Administration Medical College and ${ }^{3}$ Vajira Hospital, Bangkok, Thailand.

Dr. Alan E. Donnenfeld, MD, Center for Genetics, Maternal and Fetal, Medicine, Society Hill, 819 Locust Street, Philadelphia, PA 19107.

Received: February 1, 2005.

Accepted: March 28, 2005.

DOI: 10.1097/01.GIM.0000170774.86075.12 with pleural effusions. ${ }^{3}$ Most series of prenatally diagnosed pleural effusions involve relatively small numbers of cases. A direct comparison of the aneuploidy rate between fetuses with isolated pleural effusions and those with other congenital anomalies or significant ultrasound findings has not, to our knowledge, been previously performed. This information may be valuable in providing genetic counseling and prognostic information to couples with a fetus prenatally diagnosed with a pleural effusion. We present our data involving the chromosome analysis of 246 fetuses with pleural effusions detected on prenatal ultrasonography to determine the factors associated with an increased risk of aneuploidy, the prevalence of chromosomal abnormalities and to compare the aneuploidy rate between fetuses with isolated pleural effusions and those with pleural effusions associated with other abnormalities. To our knowledge, this is the largest series of prenatally diagnosed pleural effusions reported.

\section{MATERIALS AND METHODS}

A systematic review was performed of the Genzyme Genetics database for samples submitted from October 1994 to April 2003 with an indication of fetal pleural effusion. Clinical indications for prenatal chromosome analysis, karyotype results, gestational age at the time of specimen collection, and type of specimen (amniotic fluid, fetal blood, cystic hygroma fluid, pleural fluid) were obtained. Cases were considered isolated 
pleural effusions if the only designation on the sonographic findings indication field of the requisition form that accompanied the sample was "pleural effusion." Specimens submitted in which there were additional written findings listed on the sonographic indication field besides pleural effusion were considered as pleural effusions associated with other abnormalities. Samples submitted with the referral indication of hydrops (without a specific notation of pleural effusion) were not included in the analysis. In these cases it was unclear if the hydrops involved compartments such as amniotic fluid, subcutaneous edema, pericardial effusions, and ascites and not pleural effusions. Other indications for an amniocentesis procedure such as advanced maternal age or positive multiple marker screen were not tabulated. Pregnancy outcome information was not available.

A chi-square test was used to compare the aneuploidy rate between fetuses with isolated pleural effusion and those with additional abnormalities. Statistical significance was defined as $P<0.05$.

\section{RESULTS}

Two hundred and forty-six samples were included in the study. Most of these samples were amniotic fluid (230 cases, $93.5 \%$ ). Fluid from cystic hygromas (7 cases), pleural effusion fluid ( 7 cases), and fetal blood ( 2 cases) constituted the remaining 16 prenatal diagnosis samples. Median gestational age at which specimens were collected was 20 weeks (range 10 to 37 weeks). There were eight first trimester samples submitted and five were aneuploid. There were 94 cases $(38.5 \%$; $95 \%$ confidence interval, 32.4-44.5) of isolated pleural effusion and 152 cases $(61.5 \%$; 95\% confidence interval, 55.5- 67.6) of pleural effusions associated with additional sonographic abnormalities. Of the 152 fetuses with additional findings, there were 88 with a single additional abnormality and 64 cases with multiple additional abnormalities (Table 1). The designations utilized on Table 1 are those provided by the referring physician. Twenty-four fetuses were identified as having a pleural effusion associated with either hydrops or anasarca without further characterization as to the other sites of excess fluid accumulation. As hydrops is defined as excess fluid accumulation in more than one fetal compartment, those fetuses identified with polyhydramnios, pericardial effusion, skin edema, or ascites would all be considered as hydropic. Cystic hygroma was the most common additional anomaly identified followed by hydrops or anasarca, ascites, thickened nuchal fold, polyhydramnios, and cardiac defects.

There were nine specimens ( 2 isolated pleural effusions and 7 involving additional anomalies) in which chromosomal analysis could not be successfully accomplished. All were due to failed cell growth in culture. Of the samples that did not grow, two were cystic hygroma fluid and one was amniotic fluid from a stillborn of unknown duration. Five of the seven cystic hygroma samples yielded cytogenetic results as did all seven pleural fluid samples and both fetal blood specimens. Among the 237 samples in which karyotypes were obtained,
Table 1

Sonographic findings in 152 fetuses with nonisolated pleural effusions

\begin{tabular}{|c|c|}
\hline Abnormalities & Number of cases \\
\hline Cystic hygroma & 56 \\
\hline Hydrops fetalis or anasarca & 28 \\
\hline Ascites & 27 \\
\hline Thickened nuchal fold & 21 \\
\hline Polyhydramnios & 15 \\
\hline Cardiac defects & 13 \\
\hline Echogenic bowel & 8 \\
\hline Clubbed feet & 5 \\
\hline Renal anomalies & 5 \\
\hline Pericardial effusion & 4 \\
\hline $\begin{array}{l}\text { Others, e.g. choroid plexus cyst, short femur, } \\
\text { omphalocele, etc. }\end{array}$ & $<5$ each \\
\hline
\end{tabular}

chromosomal abnormalities were found in 84 cases $(35.3 \%$, $95 \%$ confidence interval, $29.2-41.4 \%$ ). The distribution of chromosomal findings is shown in Table 2.

Eleven chromosomal abnormalities were identified in the isolated pleural effusion group (aneuploidy rate 12\%, 95\% confidence interval, 5.3-18.6\%). These included six cases of trisomy 21, two unbalanced translocations, one case of trisomy 18 , one mosaic trisomy 20 , and one pericentric inversion of chromosome 16. There were 73 chromosomal abnormalities in fetuses with pleural effusions and additional anomalies ( $50 \%, 95 \%$ confidence interval, 41.9 to $58.1 \%$ ). The difference in aneuploidy rate between the isolated pleural effusions and the nonisolated pleural effusions was statistically significant $(P$ $<0.001)$. There were no cases of Turner syndrome $(45, \mathrm{X})$ associated with an isolated pleural effusion whereas $25.3 \%$ (37 in 145) of fetuses with pleural effusions and other abnormalities had 45,X. Figure 1 graphically illustrates the distribution of chromosome findings in both groups.

There were 22 cases $(8.9 \%)$ submitted with a written referral indication that included both an abnormal serum screen as

Table 2

Chromosomal findings among 238 informative specimens from fetuses with pleural effusions

\begin{tabular}{lc}
\hline Karyotype & Number of cases (\%) \\
\hline $46, \mathrm{XX}$ & $88(36 \%)$ \\
$46, \mathrm{XY}$ & $66(27 \%)$ \\
$45, \mathrm{X}$ & $37(15 \%)$ \\
Trisomy 21 & $31(13 \%)$ \\
Trisomy 18 & $6(2.4 \%)$ \\
Others & $10(4.1 \%)$ \\
\hline
\end{tabular}




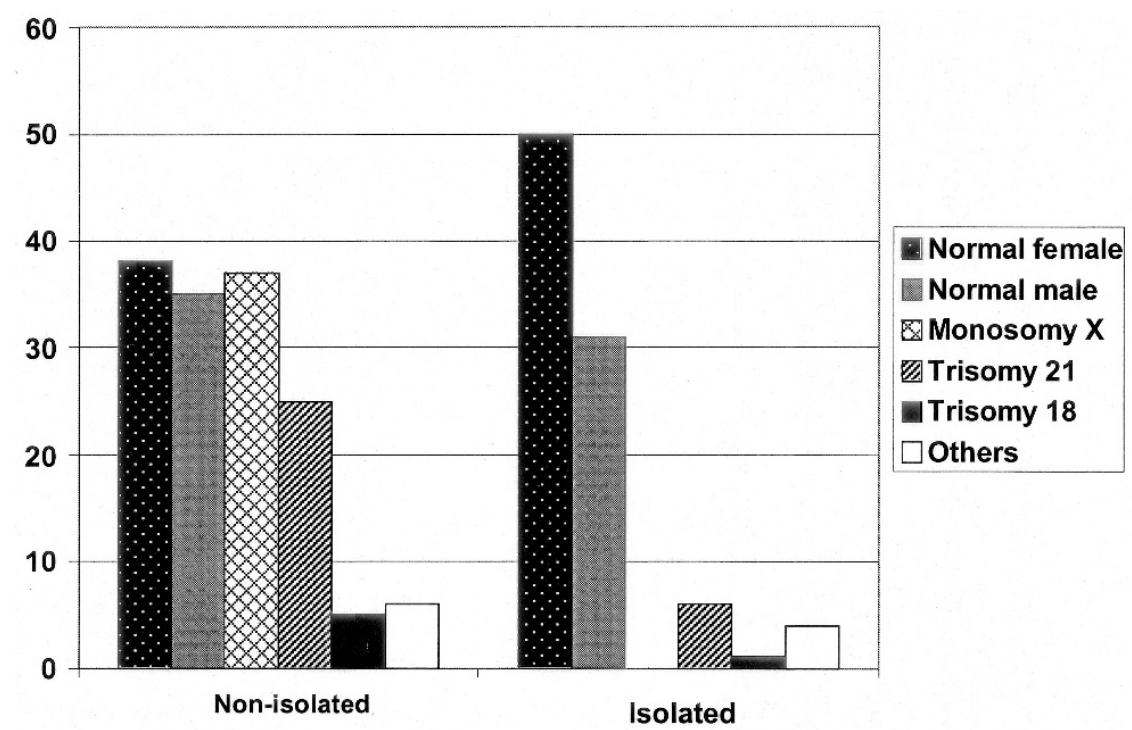

Fig. 1. Distribution of chromosomal findings in cases with isolated pleural effusion compared to pleural effusions accompanied by additional abnormalities (nonisolated)

well as a pleural effusion. Sixteen of these patients had abnormal chromosome results; nine were $45, \mathrm{X}$, four were trisomy 21 and two were trisomy 18 . All but three of these cases had additional ultrasound findings in conjunction with pleural effusions.

\section{DISCUSSION}

The prenatal diagnosis of a pleural effusion carries a poor prognosis. About half of these fetuses do not survive. ${ }^{1}$ The presence of hydrops and the detection of a pleural effusion at an early gestational age are additional factors that negatively affect survival. ${ }^{1,4}$ Whether bilateral effusions confer a worse prognosis than unilateral fluid accumulation in the fetal chest is controversial. Some investigators believe that bilateral effusions adversely affect survival, ${ }^{1}$ whereas other investigators have reported that bilaterality has no effect on outcome. ${ }^{4}$ In the current study, insufficient information on laterality was available to address this issue. Mortality is often due to pulmonary hypoplasia caused by external fluid compression of the fetal lung that results in impaired pulmonary development. Pulmonary hypoplasia can develop in as high as $30 \%$ of affected fetuses. ${ }^{6}$ Since the advent of intrauterine therapy, including fetal thoracentesis and thoracoamniotic shunting, the mortality rate has improved, ${ }^{4,7-9}$ especially in fetuses with isolated pleural effusions and normal chromosome complements. It is believed that drainage of the fetal pleural effusion may prevent associated complications such as hydrops, cardiac compression, and pulmonary hypoplasia. However, thoracentesis should be reserved for chromosomally normal fetuses with evidence of deterioration based on an increasing effusion size, cardiac compression, or hydrops. ${ }^{7,10}$ Nine percent of fetal pleural effusions have been reported to resolve spontaneously with no clinically significant adverse outcome. ${ }^{5}$
The risk of chromosome abnormalities in second and third trimester fetuses with pleural effusions has been described in several series. ${ }^{11-14}$ A meta-analysis that reviewed the English literature and collected 124 cases of fetal pleural effusion from 38 reports found that the risk of aneuploidy was 3.2\%. $\mathrm{An}$ other review reported on 82 second and third trimester fetuses with isolated pleural effusions from 31 citations in the medical literature and found a $4.9 \%$ risk of Down syndrome. ${ }^{5} \mathrm{~A}$ report by Achiron et al. ${ }^{2}$ added 29 more cases and concluded that the risk of aneuploidy in a second or third trimester fetus with a pleural effusion was $5.8 \%$.

In the present study, the prevalence of chromosome abnormalities among fetuses with isolated pleural effusions was $12 \%$, and the overall prevalence of chromosomal abnormalities in all fetuses with prenatally detected pleural effusions was 35.3\% (95\% confidence interval, 29.2-41.4). This number is greater than that reported in many other previous series involving second and third trimester fetuses. ${ }^{2,4,5}$ This difference may be due to a greater percentage of fetuses with additional (and often severe) anomalies in our study, including hydropic fetuses that had a pleural effusion as one of the findings. In addition, inclusion of eight first trimester samples that are known to be at higher risk for aneuploidy (in this series 63\%) would increase the chromosomal abnormality rate. As ascertainment was based on submission of a fetal sample for prenatal cytogenetic analysis, the principal concern was to determine the chromosome status of the fetus and not to solely determine which fetuses would be candidates for in utero pleural-amniotic shunting. When examining cases with isolated pleural effusion, the risk of fetal aneuploidy (12\%) is similar to previously reported series.

Twenty-two cases involved fetuses with both an abnormal maternal serum screen and a fetal pleural effusion detected on ultrasound examination. The risk of aneuploidy in this subset 
of patients was 19/22 (86\%). It appears that if an abnormal maternal serum screen was coupled with a sonographically identified fetal pleural effusion, the likelihood of a chromosome abnormality increased dramatically.

However, given our small sample of available maternal serum data, this should be considered a preliminary observation regarding the likelihood of aneuploidy when a patient presents with an abnormal serum screen and a fetal pleural effusion.

Among first trimester pleural effusion cases identified at 7 to 10 weeks gestation, a very high risk of an abnormal karyotype $(82 \%)$ and of spontaneous loss $(86 \%)$ has been identified. ${ }^{3}$ The authors concluded that the presence of a pleural effusion in the first trimester may be an early sign of impending fetal hydrops and subsequent demise. The vast majority of these losses were explained by chromosome abnormalities, especially $45, \mathrm{X}$, the most common aneuploidy among spontaneous losses. Our data confirms this observation as five of eight first trimester samples analyzed were chromosomally abnormal (63\%) and three of these were 45 ,X. The risk of aneuploidy associated with a first trimester diagnosis of a pleural effusion (63\%) was much greater than the frequency of aneuploidy in second and third trimester specimens (35\%), but this did not reach statistical significance by Fisher exact test $(P=0.13)$.

A concern in our study design was our reliance on data noted on the cytogenetic requisition form that accompanied the sample. It is possible that individuals who submitted the requisition forms may have completed them incorrectly or failed to include additional anomalies found in conjunction with a pleural effusion. This could result in some fetuses with multiple anomalies including a pleural effusion being classified as isolated pleural effusion cases. We attempted to contact the referring physicians who submitted these samples and obtain their ultrasound reports to verify whether the pleural effusion was isolated or not. Our efforts were unsuccessful in virtually all cases as physician offices were reluctant to share this medical information with us predominantly due to legal and confidentiality concerns. That our data revealed a $12 \%$ aneuploidy rate in the isolated pleural effusion cases and a 50\% chromosome abnormality rate in those fetuses with multiple anomalies including a pleural effusion favors the conclusion that the ultrasound information provided on the requisition form was accurate. In addition, most samples were submitted by maternal-fetal medicine specialists with expertise in ultrasonography, another indication that the information provided on the specimen requisition form was likely correct. In addition, it would be expected that if mislabeling of the requisition form occurred, it would likely involve a failure to document the presence of a fetal pleural effusion and this would result in an under-ascertainment of pleural effusion cases.

It is interesting that there were 37 cases of $45, \mathrm{X}$ detected among those fetuses with multiple anomalies including pleural effusion (the most prevalent chromosome abnormality in this group). A 45,X chromosome complement was never observed in a fetus with an isolated pleural effusion. To our knowledge, 45 , X has been diagnosed only once in a second trimester fetus with an isolated pleural effusion. ${ }^{15}$ Turner syndrome has been diagnosed in many first trimester fetuses with isolated pleural effusions. ${ }^{3,14}$ It is known that at least $98 \%$ of pregnancies with a 45,X karyotype die in utero and that this chromosome abnormality is commonly associated with cystic hygroma. In our series, cystic hygroma occurred in conjunction with fetal pleural effusion in 56 cases. This suggests that the etiology of cystic hygromas and pleural effusions are similar, likely due to lymphatic channel maldevelopment. It is also possible that pleural effusions may result as a secondary complication after the development of a cystic hygroma in fetuses with Turner syndrome. For genetic counseling purposes, this data can be used to reassure expectant parents carrying a fetus with an isolated pleural effusion that it is unlikely to be affected with a $45, \mathrm{X}$ karyotype.

Sixteen alternative fetal fluid samples (other than amniotic fluid) were submitted. There were seven cystic hygroma fluid specimens, seven pleural fluid specimens, and two fetal blood samples. There were two failed cytogenetic analyses among the seven cystic hygroma fluid samples. The success rate of chromosome analysis from cystic hygroma fluid has been found to be $76 \%$ when samples of at least $5 \mathrm{~mL}$ are submitted. ${ }^{15}$ Chromosome analysis from pleural fluid was successful in all seven samples in this series. Both the cystic hygroma and pleural fluid samples were handled identically as amniotic fluid specimens. Some investigators have advocated setting up these samples in a fashion similar to what would be performed on peripheral lymphocytes. The rationale is that cystic hygromas and pleural effusions (especially if derived from a chylothorax) may contain a predominance of lymphocytes. ${ }^{16}$ When performed in this manner on two pleural effusion specimens, results were successful in both, and available in 3 to 4 days. The current series of seven chromosome analyses from pleural fluid specimens is, to our knowledge, the largest such a series reported. All seven samples were set up as fibroblast cultures (identical to amniotic fluid cultures) and yielded good cell growth and successful cytogenetic analysis.

In summary, numerous factors affect prognosis after the prenatal diagnosis of a fetal pleural effusion. The presence of additional anomalies, the finding of an abnormal serum screen conferring an increased risk of aneuploidy, and an early gestational age at diagnosis appear to be the three most important in identifying an increased risk of aneuploidy. The presence of hydrops, the severity of the effusion, any direct effect on fetal cardiac compression, and the likelihood of the effusion leading to pulmonary hypoplasia should also be considered. Chromosome analysis is warranted in all cases of prenatally diagnosed pleural effusions. If a positive serum screen is identified in conjunction with a fetal pleural effusion, the risk of aneuploidy was $86 \%$ in our series. The presence of additional anomalies in conjunction with the pleural effusion resulted in an aneuploidy rate of 50\%. The most common chromosome abnormality in isolated fetal pleural effusions is trisomy 21. In our series, the risk of Down syndrome among isolated fetal pleural effusions was $6.3 \%$. Turner syndrome was not identified in any fetus with an isolated pleural effusion. Among fetuses with pleural effusions and additional anomalies, the risk of aneu- 
ploidy was $50 \%$ and the most frequent chromosome abnormality was $45, \mathrm{X}$. This information is valuable in providing counseling to an expectant couple after the prenatal diagnosis of a fetal pleural effusion.

\section{References}

1. Longaker MT, Laberge JM, Dansereau J, Langer JC, Cromblehome TM, Callen PW et al. Primary fetal hydrothorax: natural history and management. J Pediatr Surg 1989;24:573-576.

2. Achiron R, Weissman A, Lipitz S, Mashiach S, Goldman B. Fetal pleural effusion: the risk of fetal trisomy. Gynecol Obstet Invest 1995;39:153-156.

3. Hashimoto K, Shimizu T, Fukuda M, Ozaki M, Shimoya K, Koyama M et al. Pregnancy outcome of embryonic/fetal pleural effusion in the first trimester. J Ultrasound Med 2003;22:501-505.

4. Weber AM, Philipson EH. Fetal pleural effusion: a review and meta-analysis for prognostic indicators. Obstet Gynecol 1992;79:281-286.

5. Hagay Z, Reece A, Roberts A, Hobbins JC. Isolated fetal pleural effusion: a prenatal management dilemma. Obstet Gynecol 1993;81:147-152.

6. Estroff J, Parad R, Frigoletto F et al. The natural history of fetal hydrothorax. Ultrasound Obstet Gynecol. 1992;2:162-165.
7. Benacerraf BR, Frigoletto FD, Wilson M. Successful mid-trimester thoracentesis with analysis of the lymphocyte population in the pleural effusion. Am J Obstet Gynecol 1986;155:398-399.

8. Roberts AB, Clarkson PM, Pattison NS, Jamieson MG, Mok PM. Fetal hydrothorax in the second trimester of pregnancy: successful intrauterine at 24 weeks gestation. Fetal Ther 1986;1:203-209.

9. Nicolaides KH, Azar GB. Thoraco-amniotic shunting. Fetal Diagn Ther 1990;5:153164.

10. Petres R, Redwine F, Cruikshank D. Congenital bilateral chylothorax. JAMA 1982; 248:1360-1361.

11. Nicolaides $\mathrm{KH}$, Rodeck $\mathrm{CH}$, Gosden CM. Rapid karyotyping in nonlethal fetal malformations. Lancet 1986;1:283-286.

12. Blott M, Nicolaides KH, Greenough A. Pleuroamniotic shunting for decompression of fetal pleural effusion. Obstet Gynecol 1988;71:798-800.

13. Rodeck CH, Fisk NM, Fraser DF. Long-term in-utero drainage of fetal hydrothorax. N Engl J Med 1988;310:1135-1138.

14. Cadgin A, Pergament E. Bilateral pleural effusion at 8.5 weeks' gestation with Down syndrome and Turner syndrome. Prenat Diagn 1993;13: 659-660.

15. Donnenfeld AE, Lockwood D, Lamb A. Prenatal diagnosis from cystic hygroma fluid: The value of fluorescence in situ hybridization. Am J Obstet Gynecol 2001;185: 1004-1008.

16. Costa D, Borrell A, Margarit E, Carrio A, Soler A, Balmes I et al. Rapid fetal karytype from cystic hygroma and pleural effusion. Prenat Diagn 1995;15:141-148. 